\title{
Lycopene inhibits the cell proliferation and invasion of human head and neck squamous cell carcinoma
}

\author{
MIN YE ${ }^{1,2^{*}}$, QUNDAN WU ${ }^{1}$, MIN ZHANG ${ }^{1}$ and JINBEI HUANG ${ }^{3 *}$ \\ ${ }^{1}$ Department of Biopharmacy, School of Pharmacy, Fujian Medical University; \\ ${ }^{2}$ Fuijan Provincial Key Laboratory of Natural Medicine Pharmacology; ${ }^{3}$ Department of Urology, \\ The First Affiliated Hospital of Fujian Medical University, Fuzhou, Fujian 350004, P.R. China
}

Received June 23, 2015; Accepted July 5, 2016

DOI: $10.3892 / \mathrm{mmr} .2016 .5597$

\begin{abstract}
Lycopene has been shown to be associated with anticancer effects in numerous tumor types. However, the underlying mechanisms of lycopene in human head and neck squamous cell carcinoma (HNSCC) remain to be determined. The present study aimed to investigate the involvement of lycopene overload and the cytotoxic effects of lycopene on HNSCC cells, and to determine the possible mechanisms involved. Treatment with lycopene at a dose of $>10 \mu \mathrm{M}$ for $>24 \mathrm{~h}$ inhibited the growth of FaDu and Cal27 cells in a time- and dose-dependent manner. The clearest increase in growth inhibition was due to the apoptotic population being significantly increased. The invasion abilities decreased with $25 \mu \mathrm{M}$ lycopene exerting significant inhibitory effects $(\mathrm{P}<0.01)$. Mechanistic studies revealed that lycopene induced the upregulation of the pro-apoptotic protein, B-cell lymphoma-associated $\mathrm{X}$ protein, and therefore, resulted in the inhibition of the protein kinase $\mathrm{B}$ and mitogen-activated protein kinase signaling pathway. These data provided insights into the antitumor activity of lycopene in HNSCC cells.
\end{abstract}

\section{Introduction}

Head and neck squamous cell carcinoma (HNSCC) is a highly aggressive cancer type, with $>400,000$ new cases and 200,000 mortalities worldwide per year (1,2). Current treatment of HNSCC includes surgery, chemotherapy, radiation therapy, targeted therapy or a combination of treatments; however, patients often suffer recurrences or distant metastases, and the 5 year overall survival rate of HNSCC remains

Correspondence to: Professor Min Ye, Department of Biopharmacy, School of Pharmacy, Fujian Medical University, 1 Xue Yuan Road, University Town, Fuzhou, Fujian 350004, P.R. China E-mail: minyefj@163.com

${ }^{*}$ Contributed equally

Key words: lycopene, HNSCC, cell proliferation, cell invasion very poor, particularly for the subgroup with an advanced stage at diagnosis (3-5). The poor clinical outcome of HNSCC is largely due to recurrence and metastasis at adjacent or distant regions $(3,6)$. Although recent advances in molecular biology, cellular biology and genomics have provided insight into the molecular pathogenesis of HNSCC, the fundamental molecular mechanisms remain to be fully understood.

Lycopene (LP), a pivotal biological compound in tomatoes, has received tremendous attention as potential candidate for cancer therapy. Numerous epidemiological studies have indicated an inverse association between dietary supplementation of LP and the risk of human cancer $(7,8)$. LP has been reported to possess a broad spectrum of tumor suppressive activities in multiple human carcinomas, including leukemia, prostate, breast, colon and lung cancer (9-13). The mechanisms by which LP exerts its anticancer effects are comprehensive and diverse, targeting multiple cell signaling pathways in the processes of cellular growth, invasion, metastasis and angiogenesis. In addition, clinical intervention studies have shown that LP supplementation in men with prostate cancer decreased serum prostate-specific antigen concentrations and inhibited tumor growth. However, little attention has been given to the role of LP in the functions of HNSCC in the literature to date.

The present study aimed to determine the anticancer effect of LP on HNSCC cells in vitro. Furthermore, the present study investigated the molecular mechanisms of LP on HNSCC cells were investigated.

\section{Materials and methods}

Cell culture and treatment. The human HNSCC cell lines, FaDu and Cal27, were obtained from the Shanghai Type Culture Collection (Shanghai, China). The cells were grown in Eagle's Minimum Essential Medium (EMEM; Gibco; Thermo Fisher Scientific, Inc., Waltham, MA, USA), supplemented with $10 \%$ (v/v) fetal bovine serum (Gibco; Thermo Fisher Scientific, Inc.), $100 \mathrm{U} / \mathrm{ml}$ penicillin sodium and $100 \mu \mathrm{g} / \mathrm{ml}$ streptomycin sulfate (HyClone, Logan, UT, USA). The cells were maintained in a humidified incubator under standard conditions $\left(5 \% \mathrm{CO}_{2}\right.$ and $95 \%$ air at $\left.37^{\circ} \mathrm{C}\right)$.

LP (Sigma-Aldrich, St. Louis, MO, USA) was dissolved in tetrahydrofurancontaining (THF) to produce a $4 \mathrm{mM}$ stock solution with minimal exposure to air and light. The cells were 
treated with the indicated concentrations of LP or matching volumes of THF for the controls.

Cell proliferation and viability assays. Cell proliferation was determined using a Cell Counting kit-8 (Dojindo Laboratories, Tokyo, Japan), according to the manufacturer's protocol and incubation at $37^{\circ} \mathrm{C}$ for $2 \mathrm{~h}$. The cells were seeded into flat-bottomed 96-well plates at a density of 2,500 cells/well and were treated with various concentrations of $\operatorname{LP}(5,10,25$ and $50 \mu \mathrm{M}$ ) or THF (vehicle) for $48 \mathrm{~h}$. The optical density was measured at $450 \mathrm{~nm}$ using the Infinite 200 Pro NanoQuant (Tecan Group, Ltd., Maennedorf, Switzerland). The data are shown as the relative values in which the luminescence at a given drug concentration was compared with that of the control THF cells. All cell survival assays were performed at least three times with triplicate samples in 96-well plates.

Colony formation assays. Colony formation assays were performed by seeding 5,000 cells/well into 6-well plates. The cells were treated with $25 \mu \mathrm{M}$ LP or THF for $48 \mathrm{~h}$. The treatment was removed and fresh medium was added. The cells were incubated for 14 days and colonies were subsequently stained with $0.5 \%$ crystal violet for $15 \mathrm{~min}$ at room temperature. Colonies comprising $>50$ cells were defined as one colony and the number of colonies in 10 randomly selected fields were quantified under a microscope (DVM6; Leica Microsystems, Inc., Buffalo Grove, IL, USA). The experiment was performed in triplicate.

Cell transwell invasion assays. For the Matrigel invasion assay, cells were harvested and resuspended in EMEM, containing $0.1 \%$ (w/v) bovine serum albumin (Sangon Biotech Co., Ltd., Shanghai, China). These cells $\left(6 \times 10^{5}\right.$ cells) were added to the inserts containing $8 \mu \mathrm{m}$ pores (Corning Co, Ltd., Corning, NY, USA) coated with $24 \mathrm{mg} / \mathrm{ml}$ Matrigel (BD Biosciences, Oxford, UK). The bottom chambers contained EMEM with $10 \%$ fetal bovine serum. LP was added into the top chambers at a final concentration of $25 \mu \mathrm{M}$. Following incubation for $36 \mathrm{~h}$, the cells on the upper surface of the membrane were removed using a cotton swab. The cells that had invaded through the membrane were fixed with $4 \%$ paraformaldehyde for $30 \mathrm{~min}$ at $25^{\circ} \mathrm{C}$ and were subsequently stained with $0.5 \%$ crystal violet for $15 \mathrm{~min}$ at $25^{\circ} \mathrm{C}$. Following staining, the cells were counted in six randomly distinct fields under a light microscope (DVM6; Leica Microsystems, Inc.) at a magnification of x100. Experiments for each cell line were performed in triplicate.

Flow cytometry. The apoptosis rate of cells was measured using an Annexin V-fluorescein isothiocyanate (FITC) apoptosis detection kit (BD Biosciences), according to the manufacturer's protocol. Briefly, the cells were treated with $25 \mu \mathrm{M}$ LP or THF for $48 \mathrm{~h}$, washed with cold phosphate-buffered saline and were subsequently stained with Annexin V-FITC and propidium iodide at room temperature for $15 \mathrm{~min}$. Apoptotic cells were analyzed immediately using a flow cytometer (FACS Calibur 95; BD Biosciences) and the data were analyzed using CellQuest 3.0 software (BD Biosciences).

Immunoblotting analysis. Immunoblotting analyses were performed with standard methods. Briefly, the total protein was extracted from cells using radioimmunoprecipitation lysis buffer (Sigma-Aldrich), containing a proteinase inhibitor cocktail (Roche Diagnostics, Basel, Switzerland). Equal quantities of protein were separated by $10 \%$ sodium dodecyl sulfate-polyacrylamide gel electrophoresis and the proteins were transferred onto polyvinylidene difluoride membrane (EMD Millipore, Billerica, MA, USA). Following blocking with $5 \%$ non-fat milk in Tris-buffered saline containing $0.1 \%$ Tween-20 at room temperature, the blots were incubated with the following antibodies overnight at $4^{\circ} \mathrm{C}$ : Rabbit anti-protein kinase B antibody (AKT; 1:1,000; cat. no. 9272S), rabbit anti-phosphorylated (p-)AKT antibody (Ser473; 1:1,000; cat. no. 4060S), rabbit anti-extracellular signal-regulated kinase antibody (ERK)1/2 (1:1,000; cat. no. 4695S), p-ERK1/2 antibody (T202/Y204; 1:1,000; cat. no. 4370S), rabbit anti-cyclin D1 antibody (1:1,000; cat. no. 2978S), rabbit anti-caspase-3 antibody (1:1,000; cat. no. 9665) and rabbit anti-cleaved caspase-9 antibody (1:1,000; cat. no. 9501), all from Cell Signaling Technology, Inc. (Danvers, MA, USA), and rabbit anti-B-cell lymphoma (Bcl)-2 antibody (1:1,000; cat. no. 10783-1-AP) and rabbit anti Bcl-2-associated $\mathrm{X}$ protein antibody (Bax; 1:1,000; cat. no. 50599-2-Ig), both from Proteintech Group, Inc, Chicago, IL, USA). The protein bands were visualized using an enhanced chemiluminescence detection kit (EMD Millipore) following incubation with goat anti-rabbit horseradish peroxidase-conjugated secondary antibody (1:5,000; Cwbiotech, Beijing, China; cat. no. CW0103S). The intensity of the bands was analyzed using Image-Pro Plus software version 6.0 (Media Cybernetics, Inc., Rockville, MD, USA).

Statistical analysis. All data is presented as the mean \pm standard deviation from at least three independent experiments. Comparisons between two groups were performed using Student's t-test. Statistical analyses were performed with SPSS 15.0 statistical software package (SPSS, Inc., Chicago, IL, USA). $\mathrm{P}<0.05$ was considered to indicate a statistically significant difference.

\section{Results}

LP suppresses HNSCC proliferation and colony formation. To determine the effect of LP on HNSCC cell growth, various concentrations of LP were used to treat FaDu and Cal27 cells for 48 h. As shown in Fig. 1A, LP inhibited the viability of $\mathrm{FaDu}$ and Cal27 cells, with significant effects detected at a concentration of $25 \mu \mathrm{M}$ LP. From this, it was decided that $25 \mu \mathrm{M}$ LP would be used for further experiments. To further characterize the time-dependent toxicities of LP, FaDu and Cal27 cells were incubated with $25 \mu \mathrm{M}$ LP. In response to THF, cell proliferation analysis demonstrated that treatment with LP caused a significant cell inhibitory effect onwards for 24, 48 and $72 \mathrm{~h}$ (Fig. 1B). In addition, a colony forming assay was used to determine whether LP suppressed the proliferation ability of $\mathrm{FaDu}$ and $\mathrm{Cal} 27$ cells. This assay revealed that colony formation was significantly inhibited by treatment with LP. These results revealed that exposure to LP causes a significant inhibition of HNSCC cell proliferation.

LP induces caspase activation in HNSCC cells. Previous studies have shown that anticancer drugs can exert antip- 
A

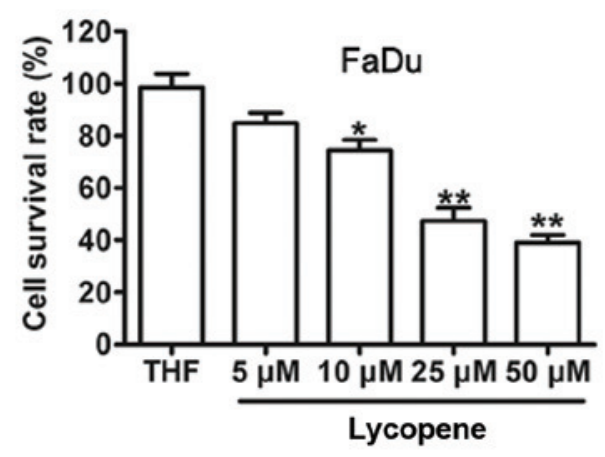

B

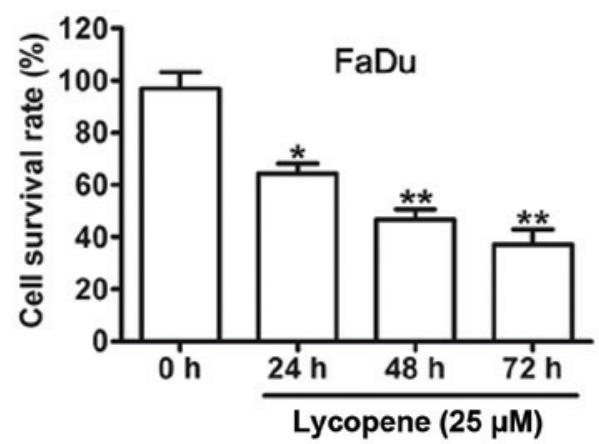

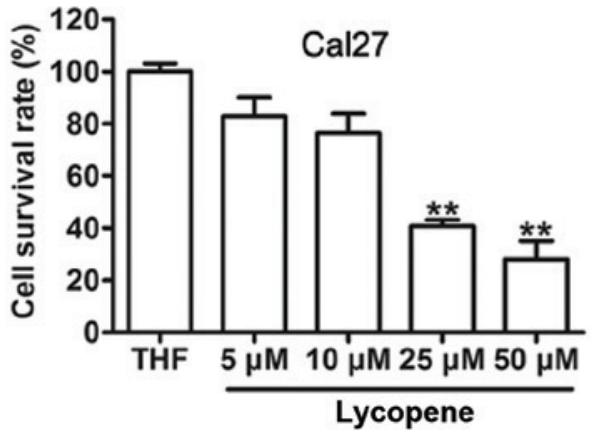

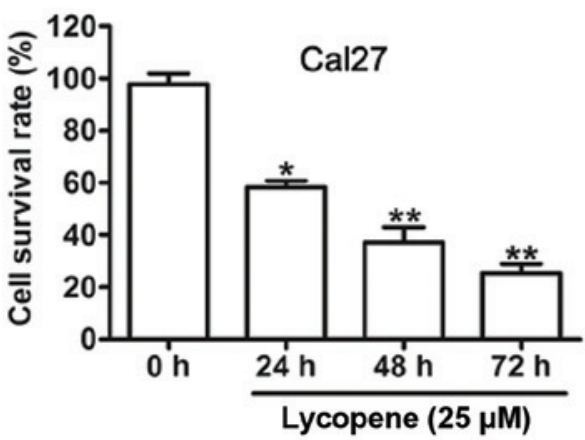

C

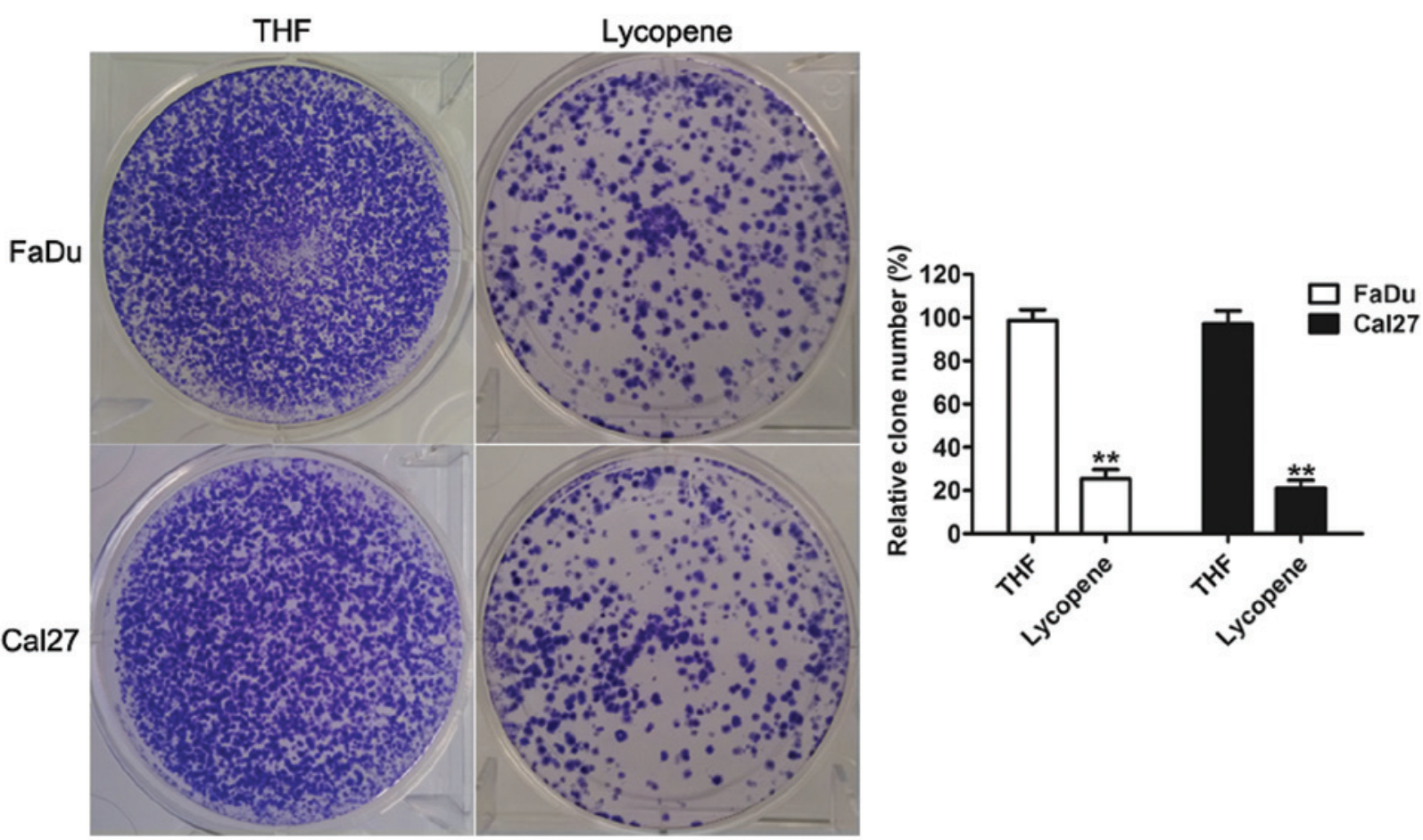

Figure 1. Effects of lycopene on the cell viability of head and neck squamous cell carcinoma. (A) The dose-dependent toxicities of lycopene on FaDu and Cal27 cells at $48 \mathrm{~h}$. (B) Cell survival was assessed in FaDu and Cal27 cells treated with $25 \mu \mathrm{M}$ lycopene for 24, 48 and $72 \mathrm{~h}$. (C) Colony formation was assessed in $\mathrm{FaDu}$ and Cal27 cells treated with THF or $25 \mu \mathrm{M}$ lycopene, and colonies were counted. The data are presented as the mean \pm standard deviation $\left(\mathrm{n}=3\right.$; ${ }^{*} \mathrm{P}<0.05$ and $^{* *} \mathrm{P}<0.01$ compared with the THF treated cells. THF, tretrahydrofurancontaining.

roliferative effects via the induction of apoptosis of tumor cells $(14,15)$. To examine the mechanisms by which LP inhibited the proliferation of HNSCC cells, the present study examined the effect of LP on the levels of apoptosis. LP significantly induced apoptosis in both $\mathrm{FaDu}$ and $\mathrm{Ca} 27$ cells (Fig. 2A). Since LP induced apoptosis, it was next determined whether the apoptosis signaling pathway was also affected by LP. As observed by western blotting, both in FaDu and Cal27 cells, LP significantly downregulated the expression of Bcl-2 (Fig. 2B), an antiapoptotic regulator. By contrast, the protein expression of Bax, caspase-3 and cleaved-caspase-9, the crucial apoptotic executioner, was increased with following 
A

THF
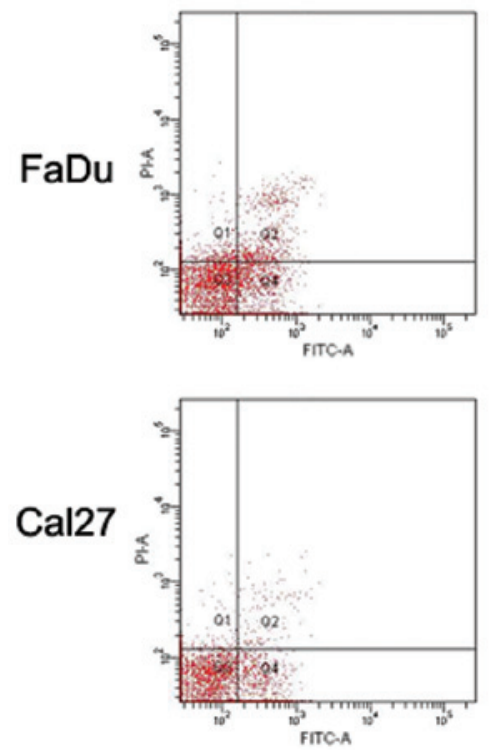

B

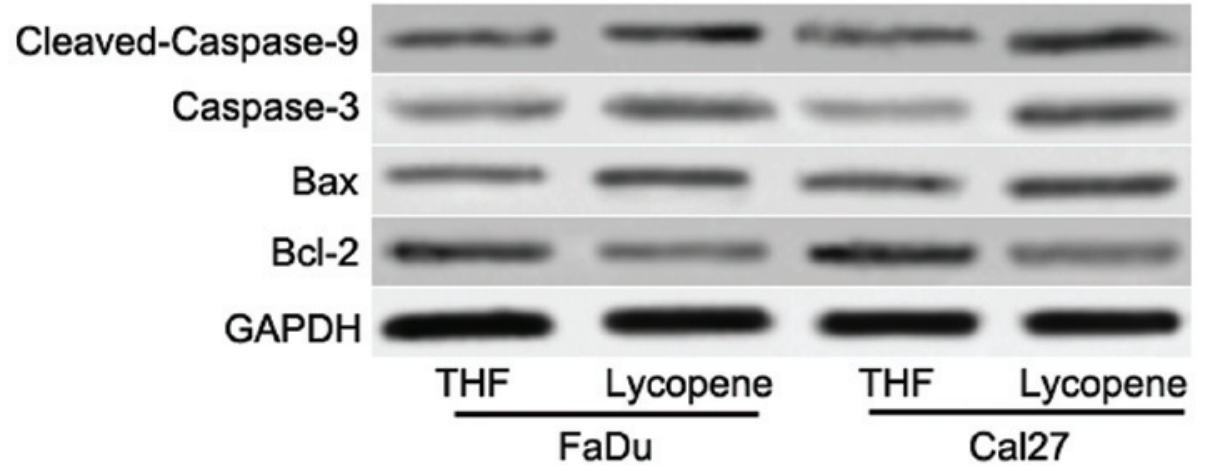

Figure 2. Lycopene induces cell apoptosis and activates the apoptosis signaling pathway in head and neck squamous cell carcinoma cells. (A) Flow cytometric analysis was performed to detect apoptotic cells. Quadrant 1 indicates dead cells, quadrant 2 indicates early apoptotic cells, quadrant 3 indicates normal cell, and quadrant 4 indicates late apoptotic cells. The data are presented as the mean \pm standard deviation $\left(\mathrm{n}=3 ;{ }^{* *} \mathrm{P}<0.01\right.$ compared with the THF treated cells) (B) Western blot analysis of the protein expression levels of Bcl-2, Bax, caspase-3 and cleaved caspase-9 in the cell lines, in response to THF or $25 \mu \mathrm{M}$ lycopene. Bcl, B-cell lymphoma; Bax, Bcl-2-associated X protein; THF, tretrahydrofurancontaining; GAPDH, glyceraldehyde 3-phosphate dehydrogenase.

treatment with LP in both FaDu and Cal27 cells (Fig. 2B). The Bax:Bcl-2 ratio highlighted the susceptibility of cells to cell death, and it was upregulated upon treatment with LP. These results suggested that LP can induce apoptosis by inhibiting the levels of Bcl-2 and by simultaneously increasing the expression levels of Bax, caspase-3 and cleaved-caspase-9.

LP suppresses the invasion of human HNSCC cells. Tumor cell migration and invasion are important in cancer progression and metastasis (16). The present study aimed to examine whether LP had a direct functional role in facilitating tumor cell invasion in HNSCC. As shown in Fig. 3, treatment with LP led to a significant decrease in cell invasion, in both FaDu and Cal27 cells, compared with THF control cells.

Cell growth and survival signaling networks are affected by $L P$. To elucidate the underlying mechanism of the sensitivity of HNSCC cells to LP, certain key signaling pathways were detected. Although numerous signaling pathways have been involved in tumorigenesis, the PI3K/AKT and MAPK signaling pathways are the most commonly assessed. Aberrant activation of PI3K/AKT and MAPK signaling pathway has been implicated in tumorigenesis and the development of a variety of tumor types, including breast, lung, ovarian, pancreatic, prostatic and gastrointestinal cancers, and HNSCC (17-21). The present study next investigated whether treatment with LP decreased the phosphorylation of AKT and ERK. After $48 \mathrm{~h}$ treatment with $25 \mu \mathrm{M}$ LP, it was revealed that LP reduced the phosphorylation of AKT and ERK (Fig. 4), however, not the total AKT and ERK levels (Fig. 4). Taken together, these data indicated that the collective inhibition of the PI3K/AKT and MAPK signaling pathways is an important feature of LP-mediated antiproliferation in HNSCC.

\section{Discussion}

Natural products, including LP from tomatoes, that exhibit the biological activity to suppress activation of cell survival pathways while selectively inducing cell death of malignant cell populations are of great importance. Numerous previous 

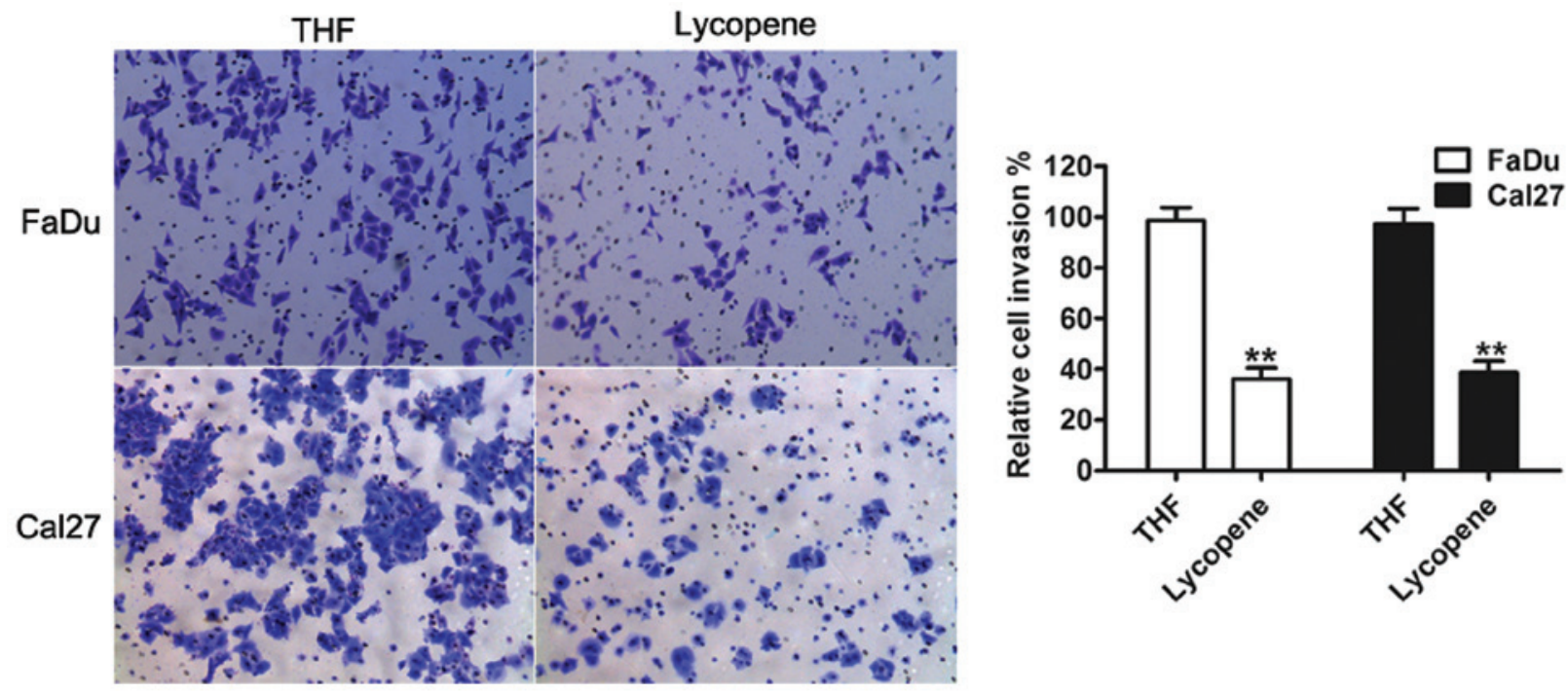

Figure 3. Effect of $25 \mu \mathrm{M}$ lycopene on the migratory properties of head and neck squamous cell carcinoma cells. A transwell invasion assay was performed to assess the migratory capacity of the FaDu and Cal27 cells in response to either THF or lycopene. Representative images of invading cells (magnification, $\mathrm{x} 100$ ) magnification. The percentage of cells migrating over the membrane were counted. The data are expressed as the mean \pm standard deviation $\left(\mathrm{n}=3\right.$; ${ }^{* *} \mathrm{P}<0.01$ compared with the THF cells. THF, tretrahydrofurancontaining.

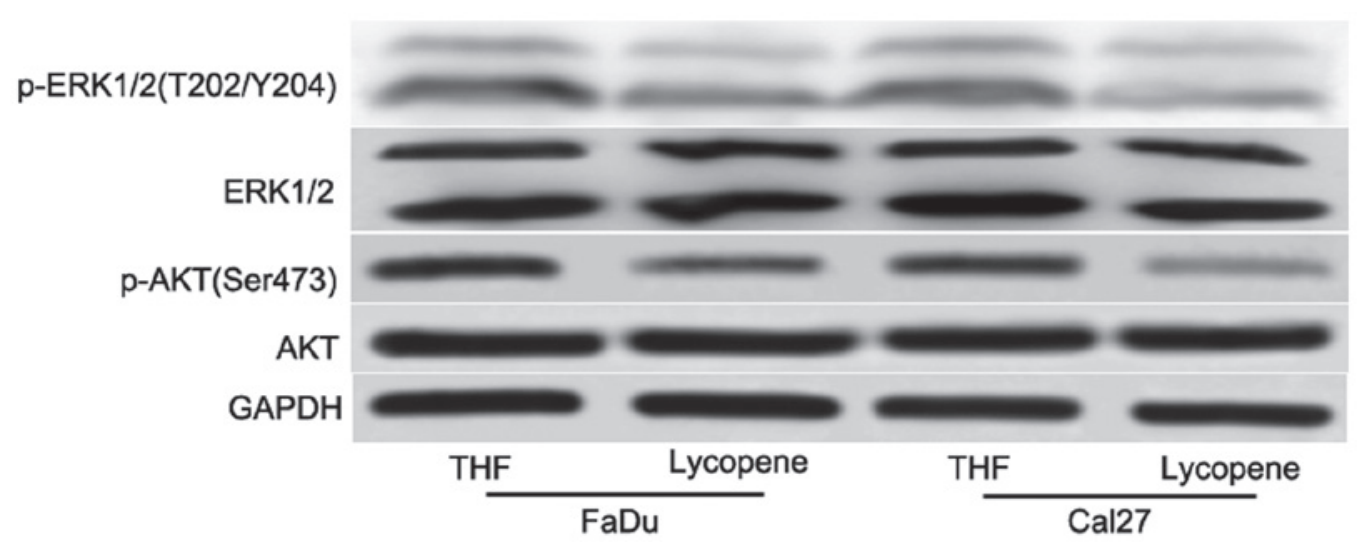

Figure 4. Lycopene inhibited pro-survival signaling pathways in head and neck squamous cell carcinoma cells. Western blot analysis of protein phosphorylation in extracts from FaDu and Cal27 cells treated for $48 \mathrm{~h}$ with $25 \mu \mathrm{M}$ lycopene or THF. THF, tretrahydrofurancontaining; p-, phosphorylated; AKT, protein kinase B; ERK, extracellular-regulated kinase; GAPDH, glyceraldehyde 3-phosphate dehydrogenase.

studies have investigated the effects of LP on cultures of human tumor cell lines and determined that LP exerts marked antiproliferative activity and triggers apoptosis $(7,11,13,14)$. In addition, it has been previously reported that ingestion of LP had preventive roles in prostate cancer $(22,23)$. Furthermore, tomato-paste extract, containing LP, induced arrest in both the G0/G1 phase and the G2/M phase of the cell cycle in prostate cancer cells via the upregulation of the expression levels of p53, p21 and p27. In the present study, in a high density culture, LP alone significantly inhibited proliferation, colony formation, induced apoptosis and decreased the invasion ability in HNSCC cells. Furthermore, it was observed that the change of apoptosis, cell growth and survival signaling stemming from LP exposure were responsible for the inhibition of the cell cycle and cell apoptosis response triggered by LP.

It was previously reported that LP was associated with cancer cell proliferation, mobility, migration and invasion in numerous cancer types $(11,13,24)$. This was most likely by inducing antiproliferative and apoptosis activities. The present study investigated and revealed that cell proliferative was suppressed, and cell apoptosis was enhanced following treatment with LP. Repression of cell growth may be due to the induction of apoptosis, as demonstrated in the present study. It was also demonstrated that Bcl-2, an antiapoptotic member of the Bcl family, was downregulation upon treatment with LP. By contrast, Bax, a pro-apoptotic protein, was induced by treatment with LP. Additionally, other pro-apoptotic signaling, including caspase 3 and caspase 9 were also significantly increased.

Previous studies have shown that treatment with LP is associated with the migration and invasion capability of cancer cells (25-27). The present study confirmed that treatment with LP inhibited HNSCC cell invasion in vitro, in association with decreased expression levels of p-AKT and p-ERK. These findings provided additional mechanistic insight into the ways in which LP inhibits the aggressive behavior of HNSCC.

In conclusion, this is the first report, to the best of our knowledge, to investigate the effects of LP on HNSCC cell lines. It 
was demonstrated that LP is a potential anticancer drug from natural products for patients with HNSCC. In vitro investigation revealed that treatment with LP inhibited cell growth, induced apoptosis and decreased the invasion activity of HNSCC cells by inducing apoptosis, and the PI3K/AKT and MAPK signaling pathways. Further studies are underway to assess the molecular mechanisms through which LP is likely to influence the antitumor properties of more cancer cells in our laboratory.

\section{References}

1. Jemal A, Bray F, Center MM, Ferlay J, Ward E and Forman D: Global cancer statistics. CA Cancer J Clin 61: 69-90, 2011.

2. Parkin DM, Bray F, Ferlay J and Pisani P: Global cancer statistics, 2002. CA Cancer J Clin 55: 74-108, 2005.

3. Leemans CR, Braakhuis BJ and Brakenhoff RH: The molecular biology of head and neck cancer. Nat Rev Cancer 11: 9-22, 2011.

4. Argiris A, Karamouzis MV, Raben D and Ferris RL: Head and neck cancer. Lancet 371: 1695-1709, 2008.

5. Tamagawa S, Beder LB, Hotomi M, Gunduz M, Yata K, Grenman R and Yamanaka N: Role of miR-200c/miR-141 in the regulation of epithelial-mesenchymal transition and migration in head and neck squamous cell carcinoma. Int J Mol Med 33: 879-886, 2014

6. Wang T, Chen T, Niu H, Li C, Xu C, Li Y, Huang R, Zhao J and Wu S: MicroRNA-218 inhibits the proliferation and metastasis of esophageal squamous cell carcinoma cells by targeting BMI1. Int J Mol Med 36: 93-102, 2015.

7. Zhang YY, Lu L, Abliz G and Mijit F: Serum carotenoid, retinol and tocopherol concentrations and risk of cervical cancer among chinese women. Asian Pac J Cancer Prev 16: 2981-2986, 2015.

8. Ansari MS and Sgupta NP: A comparison of lycopene and orchidectomy vs orchidectomy alone in the management of advanced prostate cancer. BJU Int 95: 453, 2005.

9. Gharib A and Faezizadeh Z: In vitro anti-telomerase activity of novel lycopene-loaded nanospheres in the human leukemia cell line K562. Pharmacogn Mag 10 (Suppl 1): S157-S163, 2014.

10. Ford NA, Elsen AC, Zuniga K, Lindshield BL and Erdman JW Jr: Lycopene and apo-12'-lycopenal reduce cell proliferation and alter cell cycle progression in human prostate cancer cells. Nutr Cancer 63: 256-263, 2011.

11. Takeshima M, Ono M, Higuchi T, Chen C, Hara T and Nakano S: Anti-proliferative and apoptosis-inducing activity of lycopene against three subtypes of human breast cancer cell lines. Cancer Sci 105: 252-257, 2014.

12. Tang FY, Pai MH and Wang XD: Consumption of lycopene inhibits the growth and progression of colon cancer in a mouse xenograft model. J Agric Food Chem 59: 9011-9021, 2011.
13. Palozza P, Simone RE, Catalano A and Mele MC: Tomato lycopene and lung cancer prevention: From experimental to human studies. Cancers (Basel) 3: 2333-2357, 2011.

14. Holzapfel NP, Holzapfel BM, Champ S, Feldthusen J, Clements J and Hutmacher DW: The potential role of lycopene for the prevention and therapy of prostate cancer: From molecular mechanisms to clinical evidence. Int J Mol Sci 14: 14620-14646, 2013.

15. Goldar S, Khaniani MS, Derakhshan SM and Baradaran B: Molecular mechanisms of apoptosis and roles in cancer development and treatment. Asian Pac J Cancer Prev 16: 2129-2144, 2015.

16. Hanahan D and Weinberg RA: Hallmarks of cancer: The next generation. Cell 144: 646-674, 2011.

17. Wheeler SE, Suzuki S, Thomas SM, Sen M, Leeman-Neill RJ, Chiosea SI, Kuan CT, Bigner DD, Gooding WE, Lai SY and Grandis JR: Epidermal growth factor receptor variant III mediates head and neck cancer cell invasion via STAT3 activation. Oncogene 29: 5135-5145, 2010.

18. Zuo T, Liu TM, Lan X, Weng YI, Shen R, Gu F, Huang YW, Liyanarachchi S, Deatherage DE, Hsu PY, et al: Epigenetic silencing mediated through activated PI3K/AKT signaling in breast cancer. Cancer Res 71: 1752-1762, 2011.

19. da Silva HB, Amaral EP, Nolasco EL, de Victo NC, Atique R, Jank CC, Anschau V, Zerbini LF and Correa RG: Dissecting major signaling pathways throughout the development of prostate cancer. Prostate Cancer 2013: 920612, 2013.

20. Shi L, Wang L, Wang B, Cretoiu SM, Wang Q, Wang X and Chen C: Regulatory mechanisms of betacellulin in CXCL8 production from lung cancer cells. J Transl Med 12: 70, 2014.

21. Manning BD and Cantley LC: AKT/PKB signaling: Navigating downstream. Cell 129: 1261-1274, 2007.

22. Giovannucci E, Ascherio A, Rimm EB, Stampfer MJ, Colditz GA and Willett WC: Intake of carotenoids and retinol in relation to risk of prostate cancer. J Natl Cancer Inst 87: 1767-1776, 1995.

23. Wertz K: Lycopene effects contributing to prostate health. Nutr Cancer 61: 775-783, 2009.

24. Aras A, Khokhar AR, Qureshi MZ, Silva MF, Sobczak-Kupiec A Pineda EA, Hechenleitner AA and Farooqi AA: Targeting cancer with nano-bullets: Curcumin, EGCG, resveratrol and quercetin on flying carpets. Asian Pac J Cancer Prev 15: 3865-3871, 2014.

25. Chan CM, Fang JY, Lin HH, Yang CY and Hung CF: Lycopene inhibits PDGF-BB-induced retinal pigment epithelial cell migration by suppression of PI3K/Akt and MAPK pathways. Biochem Biophys Res Commun 388: 172-176, 2009.

26. Elgass S, Cooper A and Chopra M: Lycopene treatment of prostate cancer cell lines inhibits adhesion and migration properties of the cells. Int J Med Sci 11: 948-954, 2014

27. Huang CS, Shih MK, Chuang CH and Hu ML: Lycopene inhibits cell migration and invasion and upregulates $\mathrm{Nm} 23-\mathrm{H} 1$ in a highly invasive hepatocarcinoma, SK-Hep-1 cells. J Nutr 135: 2119-2123, 2005. 